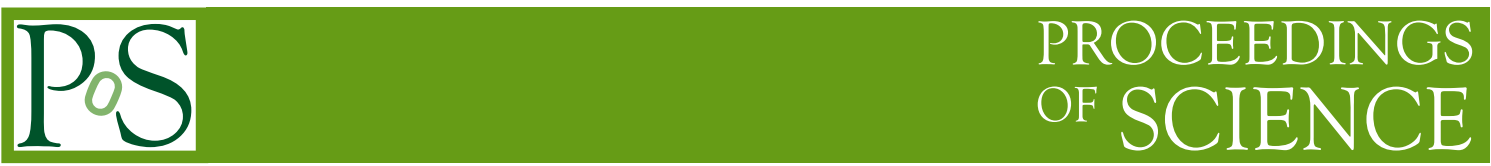

\title{
Exotic hadrons in Experiment and on the Lattice
}

\author{
John Bulava* \\ Trinity College, Dublin \\ E-mail: jbulava@maths.tcd.ie
}

\begin{abstract}
Although the constituent quark model correctly predicts the quantum numbers of nearly all known hadrons, it fails to account for so-called 'exotic' states. After briefly reviewing the current experimental status of some candidate exotic hadrons, I will discuss prospects for elucidation of their properties using numerical Lattice QCD simulations. Recent theoretical advances have greatly improved lattice calculations of hadronic resonances, but some of the most interesting systems (such as exotic charmonium states) remain a challenge.
\end{abstract}

Frontiers of Fundamental Physics 14 - FFP14,

15-18 July 2014

Aix Marseille University (AMU) Saint-Charles Campus, Marseille

\footnotetext{
*Speaker.
} 